\title{
Anti-Invariant Semi-Riemannian Submersions from Almost Para-Hermitian Manifolds
}

\author{
Yılmaz Gündüzalp \\ Department of Mathematics, Dicle University, 21280 Diyarbakır, Turkey \\ Correspondence should be addressed to Yllmaz Gündüzalp; zygunduzalp@gmail.com
}

Received 21 July 2013; Accepted 4 October 2013

Academic Editor: Gen-Qi Xu

Copyright (C) 2013 Yılmaz Gündüzalp. This is an open access article distributed under the Creative Commons Attribution License, which permits unrestricted use, distribution, and reproduction in any medium, provided the original work is properly cited.

We introduce anti-invariant semi-Riemannian submersions from almost para-Hermitian manifolds onto semi-Riemannian manifolds. We give an example, investigate the geometry of foliations which are arisen from the definition of a semi-Riemannian submersion, and check the harmonicity of such submersions. We also obtain curvature relations between the base manifold and the total manifold.

\section{Introduction}

The theory of Riemannian submersion was introduced by O'Neill and Gray in $[1,2]$, respectively. Later, Riemannian submersions were considered between almost complex manifolds by Watson in [3] under the name of almost Hermitian submersion. He showed that if the total manifold is a Kähler manifold, then the base manifold is also a Kähler manifold. Since then, Riemannian submersions have been used as an effective tool to describe the structure of a Riemannian manifold equipped with a differentiable structure. Presently, there is an extensive literature on the Riemannian submersions with different conditions imposed on the total space and on the fibres. For instance, Riemannian submersions between almost contact manifolds were studied by Chinea in [4] under the name of almost contact submersions. Riemannian submersions have been also considered for quaternionic Kähler manifolds [5] and para-quaternionic Kähler manifolds [6, 7]. This kind of submersions have been studied with different names by many authors (see [8-14], and more).

On the other hand, para-complex manifolds, almost para-Hermitian manifolds, and para-Kähler manifolds were defined by Libermann [15] in 1952. In fact, such manifolds arose in [16]. Indeed, Rashevskij introduced the properties of para-Kähler manifolds, when he considered a metric of signature $(m, m)$ defined from a potential function the so-called scalar field on a $2 m$-dimensional locally product manifold called by him stratified space.
Semi-Riemannian submersions were introduced by O'Neill in his book [17]. It is known that such submersions have their applications in Kaluza-Klein theories, Yang-Mills equations, strings, and supergravity. For applications of semi-Riemannian submersions, see [18]. Since almost paraHermitian manifolds are semi-Riemannian manifolds, one should consider semi-Riemannian submersions between such manifolds.

The paper is organized as follows. In Section 2 we recall some notions needed for this paper. In Section 3 we give the definition of anti-invariant semi-Riemannian submersions, provide an example and investigate the geometry of leaves of the distributions. We give necessary and sufficient conditions for such submersions to be totally geodesic or harmonic. We also find necessary and sufficient conditions for a Lagrangian semi-Riemannian submersion, a special anti-invariant semiRiemannian submersion, to be totally geodesic. Moreover, we obtain decomposition theorems for the total manifold of such submersions. Finally, we obtain curvature relations between the base manifold and the total manifold.

\section{Preliminaries}

In this section, we define almost para-Hermitian manifolds, recall the notion of semi-Riemannian submersions between semi-Riemannian manifolds, and give a brief review of basic facts of semi-Riemannian submersions. 
An almost para-Hermitian manifold is a manifold $M$ endowed with an almost para-complex structure $P \neq \pm I$ and a semi-Riemannian metric $g$ such that

$$
P^{2}=I, \quad g(P X, P Y)=-g(X, Y)
$$

for $X, Y$ tangent to $M$, where $I$ is the identity map. The dimension of $M$ is even and the signature of $g$ is $(m, m)$, where $\operatorname{dim} M=2 m$. Consider an almost para-Hermitian manifold $(M, P, g)$ and denote by $\nabla$ the Levi-Civita connection on $M$ with respect to $g$. Then $M$ is called a para-Kähler manifold if $P$ is parallel with respect to $\nabla$; that is,

$$
\left(\nabla_{X} P\right) Y=0
$$

for $X, Y$ tangent to $M$ [19].

Let $(M, g)$ and $\left(N, g^{\prime}\right)$ be two connected semi-Riemannian manifolds of index $s(0 \leq s \leq \operatorname{dim} M)$ and $s^{\prime}(0 \leq$ $\left.s^{\prime} \leq \operatorname{dim} N\right)$ respectively, with $s>s^{\prime}$. A semi-Riemannian submersion is a smooth map $\pi: M \rightarrow N$ which is onto and satisfies the following conditions:

(i) $\pi_{* p}: T_{p} M \rightarrow T_{\pi(p)} N$ is onto for all $p \in M$;

(ii) the fibres $\pi^{-1}\left(p^{\prime}\right), p^{\prime} \in N$ are semi-Riemannian submanifolds of $M$;

(iii) $\pi_{*}$ preserves scalar products of vectors normal to fibres.

The vectors tangent to the fibres are called vertical and those normal to the fibres are called horizontal. We denote by $\mathscr{V}$ the vertical distribution, by $\mathscr{H}$ the horizontal distribution and by $v$ and $h$ the vertical and horizontal projection. A horizontal vector field $X$ on $M$ is said to be basic if $X$ is $\pi$ related to a vector field $X^{\prime}$ on $N$. It is clear that every vector field $X^{\prime}$ on $N$ has a unique horizontal lift $X$ to $M$ and $X$ is basic.

We recall that the sections of $\mathscr{V}$, respectively $\mathscr{H}$, are called the vertical vector fields, respectively, horizontal vector fields. A semi-Riemannian submersion $\pi: M \rightarrow N$ determines two $(1,2)$ tensor fields $T$ and $A$ on $M$, by the formulas

$$
\begin{aligned}
& T(E, F)=T_{E} F=h \nabla_{v E} v F+v \nabla_{v E} h F, \\
& A(E, F)=A_{E} F=v \nabla_{h E} h F+h \nabla_{h E} v F
\end{aligned}
$$

for any $E, F \in \Gamma(T M)$, where $v$ and $h$ are the vertical and horizontal projections (see $[20,21]$ ). From (3), one can obtain

$$
\begin{gathered}
\nabla_{U} W=T_{U} W+\widehat{\nabla}_{U} W ; \\
\nabla_{U} X=T_{U} X+h\left(\nabla_{U} X\right) ; \\
\nabla_{X} U=v\left(\nabla_{X} U\right)+A_{X} U ; \\
\nabla_{X} Y=A_{X} Y+h\left(\nabla_{X} Y\right),
\end{gathered}
$$

for any $X, Y \in \Gamma\left(\left(\operatorname{ker} \pi_{*}\right)^{\perp}\right), U, W \in \Gamma\left(\operatorname{ker} \pi_{*}\right)$. Moreover, if $X$ is basic then $h\left(\nabla_{U} X\right)=h\left(\nabla_{X} U\right)=A_{X} U$.

We note that for $U, V \in \Gamma\left(\operatorname{ker} \pi_{*}\right), T_{U} V$ coincides with the second fundamental form of the immersion of the fibre submanifolds and for $X, Y \in \Gamma\left(\left(\operatorname{ker} \pi_{*}\right)^{\perp}\right)$,
$A_{X} Y=(1 / 2) v[X, Y]$ reflecting the complete integrability of the horizontal distribution $\mathscr{H}$. It is known that $A$ is alternating on the horizontal distribution: $A_{X} Y=-A_{Y} X$, for $X, Y \in \Gamma\left(\left(\operatorname{ker} \pi_{*}\right)^{\perp}\right)$ and $T$ is symmetric on the vertical distribution: $T_{U} V=T_{V} U$, for $U, V \in \Gamma\left(\operatorname{ker} \pi_{*}\right)$.

We now recall the following result which will be useful for later.

Lemma 1 (see $[17,21])$. If $\pi: M \rightarrow N$ is a semi-Riemannian submersion and $X, Y$ basic vector fields on $M, \pi$-related to $X^{\prime}$ and $Y^{\prime}$ on $N$, then one has the following properties:

(1) $h[X, Y]$ is a basic vector field and $\pi_{*} h[X, Y]=$ $\left[X^{\prime}, Y^{\prime}\right] \circ \pi$;

(2) $h\left(\nabla_{X} Y\right)$ is a basic vector field $\pi$-related to $\left(\nabla_{X^{\prime}}^{\prime} Y^{\prime}\right)$, where $\nabla$ and $\nabla^{\prime}$ are the Levi-Civita connection on $M$ and $N$;

(3) $[E, U] \in \Gamma\left(\operatorname{ker} \pi_{*}\right)$, for any $U \in \Gamma\left(\operatorname{ker} \pi_{*}\right)$ and for any basic vector field $E$.

Let $(M, g)$ and $\left(N, g^{\prime}\right)$ be (semi-)Riemannian manifolds and $\pi: M \rightarrow N$ is a smooth map. Then the second fundamental form of $\pi$ is given by

$$
\left(\nabla \pi_{*}\right)(X, Y)=\nabla_{X}^{\pi} \pi_{*} Y-\pi_{*}\left(\nabla_{X} Y\right)
$$

for $X, Y \in \Gamma(T M)$, where we denote conveniently by $\nabla$ the Levi-Civita connections of the metrics $g$ and $g^{\prime}$. Recall that $\pi$ is said to be harmonic if trace $\left(\nabla \pi_{*}\right)=0$ and $\pi$ is called a totally geodesic map if $\left(\nabla \pi_{*}\right)(X, Y)=0$ for $X, Y \in$ $\Gamma(T M)[22]$. It is known that the second fundamental form is symmetric.

\section{Anti-Invariant Semi-Riemannian Submersions}

In this section, we define anti-invariant semi-Riemannian submersions from a para-Kähler manifold onto a semiRiemannian manifold, investigate the integrability of distributions, and obtain a necessary and sufficient condition for such submersions to be totally geodesic map.

Definition 2. Let $(M, g, P)$ be an almost para-Hermitian manifold and $\left(N, g^{\prime}\right)$ a semi-Riemannian manifold. Suppose that there exists a semi-Riemannian submersion $\pi: M \rightarrow N$ such that $\operatorname{ker} \pi_{*}$ is anti-invariant with respect to $P$; that is, $P\left(\operatorname{ker} \pi_{*}\right) \subseteq\left(\operatorname{ker} \pi_{*}\right)^{\perp}$. Then we say $\pi$ is an anti-invariant semi-Riemannian submersion.

Let $\pi:(M, g, P) \rightarrow\left(N, g^{\prime}\right)$ be an anti-invariant semi-Riemannian submersion from a para-Kähler manifold $(M, g, P)$ to a semi-Riemannian manifold $\left(N, g^{\prime}\right)$. First of all, from Definition 2, we have $P\left(\operatorname{ker} \pi_{*}\right)^{\perp} \cap\left(\operatorname{ker} \pi_{*}\right) \neq 0$. We denote the complementary orthogonal distribution to $P\left(\operatorname{ker} \pi_{*}\right)$ in $\left(\operatorname{ker} \pi_{*}\right)^{\perp}$ by $\mu$. Then we have

$$
\left(\operatorname{ker} \pi_{*}\right)^{\perp}=P\left(\operatorname{ker} \pi_{*}\right) \oplus \mu .
$$


It is easy to see that $\mu$ is an invariant distribution of $\left(\operatorname{ker} \pi_{*}\right)^{\perp}$, under the endomorphism $P$. Thus, for $X \in$ $\Gamma\left(\left(\operatorname{ker} \pi_{*}\right)^{\perp}\right)$, we have

$$
P X=B X+C X
$$

where $B X \in \Gamma\left(\operatorname{ker} \pi_{*}\right)$ and $C X \in \Gamma(\mu)$. On the other hand, since $\pi_{*}\left(\left(\operatorname{ker} \pi_{*}\right)^{\perp}\right)=T N$ and $\pi$ is a semi-Riemannian submersion, using (10) we derive $g^{\prime}\left(\pi_{*} P V, \pi_{*} C X\right)=0$, for every $X \in \Gamma\left(\left(\operatorname{ker} \pi_{*}\right)^{\perp}\right)$ and $V \in \Gamma\left(\operatorname{ker} \pi_{*}\right)$, which implies that

$$
T N=\pi_{*}\left(P\left(\operatorname{ker} \pi_{*}\right)\right) \oplus \pi_{*}(\mu) .
$$

Note that given a semi-Euclidean space $R_{2}^{4}$ with coordinates $\left(x_{1}, \ldots, x_{4}\right)$, we can canonically choose an almost paracomplex structure $P$ on $R_{2}^{4}$ as follows:

$$
\begin{aligned}
& P\left(a_{1} \frac{\partial}{\partial x_{1}}+a_{2} \frac{\partial}{\partial x_{2}}+a_{3} \frac{\partial}{\partial x_{3}}+a_{4} \frac{\partial}{\partial x_{4}}\right) \\
& \quad=-a_{2} \frac{\partial}{\partial x_{1}}-a_{1} \frac{\partial}{\partial x_{2}}+a_{4} \frac{\partial}{\partial x_{3}}+a_{3} \frac{\partial}{\partial x_{4}},
\end{aligned}
$$

where $a_{1}, \ldots, a_{4} \in R$.

Also the neutral metric compatible with $P$ is

$$
g=\left(d x_{1}\right)^{2}-\left(d x_{2}\right)^{2}+\left(d x_{3}\right)^{2}-\left(d x_{4}\right)^{2} .
$$

We now give an example of an anti-invariant semi-Riemannian submersion.

Example 3. Let $\pi: R_{2}^{4} \rightarrow R_{1}^{2}$ be a map defined $\pi\left(x_{1}, x_{2}\right.$, $\left.x_{3}, x_{4}\right)=\left(\left(x_{1}+x_{3}\right) / \sqrt{2},\left(x_{2}+x_{4}\right) / \sqrt{2}\right)$. Then, by direct calculations

$$
\begin{aligned}
& \operatorname{ker} \pi_{*}=\operatorname{Span}\left\{Z_{1}=-\frac{\partial}{\partial x_{1}}+\frac{\partial}{\partial x_{3}}, Z_{2}=-\frac{\partial}{\partial x_{2}}+\frac{\partial}{\partial x_{4}}\right\}, \\
& \left(\operatorname{ker} \pi_{*}\right)^{\perp}=\operatorname{Span}\left\{X_{1}=\frac{\partial}{\partial x_{1}}+\frac{\partial}{\partial x_{3}}, X_{2}=\frac{\partial}{\partial x_{2}}+\frac{\partial}{\partial x_{4}}\right\} .
\end{aligned}
$$

Then it is easy to see that $\pi$ is a semi-Riemannian submersion. Moreover $P Z_{1}=X_{2}$ and $P Z_{2}=X_{1}$ imply that $P\left(\operatorname{ker} \pi_{*}\right)=$ $\left(\operatorname{ker} \pi_{*}\right)^{\perp}$. As a result, $\pi$ is an anti-invariant semi-Riemannian submersion.

Lemma 4. Let $\pi$ be an anti-invariant semi-Riemannian submersion from a para-Kähler manifold $(M, g, P)$ to a semiRiemannian manifold $\left(N, g^{\prime}\right)$. Then one has

$$
\begin{gathered}
g(C Y, P V)=0, \\
g\left(\nabla_{X} C Y, P V\right)=-g\left(C Y, P A_{X} V\right)
\end{gathered}
$$

for $X, Y \in \Gamma\left(\left(\operatorname{ker} \pi_{*}\right)^{\perp}\right)$ and $V \in \Gamma\left(\operatorname{ker} \pi_{*}\right)$.

Proof. For $Y \in \Gamma\left(\left(\operatorname{ker} \pi_{*}\right)^{\perp}\right)$ and $V \in \Gamma\left(\operatorname{ker} \pi_{*}\right)$, using (1) we have

$$
g(C Y, P V)=g(P Y-B Y, P V)=g(P Y, P V)
$$

due to $B Y \in \Gamma\left(\operatorname{ker} \pi_{*}\right)$ and $P V \in \Gamma\left(\left(\operatorname{ker} \pi_{*}\right)^{\perp}\right)$. Hence $g(P Y, P V)=-g(Y, V)=0$ which is (15). Since $M$ is a paraKähler manifold, using (15) we get

$$
g\left(\nabla_{X} C Y, P V\right)=-g\left(C Y, P \nabla_{X} V\right)
$$

for $X, Y \in \Gamma\left(\left(\operatorname{ker} \pi_{*}\right)^{\perp}\right)$ and $V \in \Gamma\left(\operatorname{ker} \pi_{*}\right)$. Then using (6) we have

$$
g\left(\nabla_{X} C Y, P V\right)=-g\left(C Y, P A_{X} V\right)-g\left(C Y, P v \nabla_{X} V\right) .
$$

Since $P \nu \nabla_{X} V \in \Gamma\left(P \operatorname{ker} \pi_{*}\right)$, we obtain (16).

We now study the integrability of the distribution $\left(\operatorname{ker} \pi_{*}\right)^{\perp}$ and then we investigate the geometry of leaves of $\operatorname{ker} \pi_{*}$ and $\left(\operatorname{ker} \pi_{*}\right)^{\perp}$. We note that it is known that the distribution $\operatorname{ker} \pi_{*}$ is integrable.

Theorem 5. Let $\pi$ be an anti-invariant semi-Riemannian submersion from a para-Kähler manifold $(M, g, P)$ to a semiRiemannian manifold $\left(N, g^{\prime}\right)$. Then the following assertions are equivalent to each other:

(i) $\left(\operatorname{ker} \pi_{*}\right)^{\perp}$ is integrable.

(ii) $g^{\prime}\left(\left(\nabla \pi_{*}\right)(X, B Y), \pi_{*} P V\right)=g^{\prime}\left(\left(\nabla \pi_{*}\right)(Y, B X)\right.$, $\left.\pi_{*} P V\right)-g\left(C Y, P A_{X} V\right)+g\left(C X, P A_{Y} V\right)$.

(iii) $g\left(P V, A_{Y} B X-A_{X} B Y\right)=-g\left(C Y, P A_{X} V\right)+$ $g\left(C X, P A_{Y} V\right)$,

for $X, Y \in \Gamma\left(\left(\operatorname{ker} \pi_{*}\right)^{\perp}\right)$ and $V \in \Gamma\left(\operatorname{ker} \pi_{*}\right)$.

Proof. For $Y \in \Gamma\left(\left(\operatorname{ker} \pi_{*}\right)^{\perp}\right)$ and $V \in \Gamma\left(\operatorname{ker} \pi_{*}\right)$, we see from Definition 2, $P V \in \Gamma\left(\left(\operatorname{ker} \pi_{*}\right)^{\perp}\right)$ and $P Y \in \Gamma\left(\operatorname{ker} \pi_{*} \oplus \mu\right)$. Thus using (1) and (2) we obtain

$$
g([X, Y], V)=-g\left(\nabla_{X} P Y, P V\right)+g\left(\nabla_{Y} P X, P V\right) .
$$

Then from (10) we get

$$
\begin{aligned}
g([X, Y], V)= & -g\left(\nabla_{X} B Y, P V\right)-g\left(\nabla_{X} C Y, P V\right) \\
& +g\left(\nabla_{Y} B X, P V\right)+g\left(\nabla_{Y} C X, P V\right) .
\end{aligned}
$$

Since $\pi$ is a semi-Riemannian submersion, we have

$$
\begin{aligned}
g([X, Y], V)= & -g^{\prime}\left(\pi_{*} \nabla_{X} B Y, \pi_{*} P V\right)-g\left(\nabla_{X} C Y, P V\right) \\
& +g^{\prime}\left(\pi_{*} \nabla_{Y} B X, \pi_{*} P V\right)+g\left(\nabla_{Y} C X, P V\right) .
\end{aligned}
$$

Thus, from (8) and (16) we obtain

$$
\begin{aligned}
g([X, Y], V)= & g^{\prime}\left(\left(\nabla \pi_{*}\right)(X, B Y)-\left(\nabla \pi_{*}\right)(Y, B X), \pi_{*} P V\right) \\
& +g\left(C Y, P A_{X} V\right)-g\left(C X, P A_{Y} V\right)
\end{aligned}
$$

which proves (i) $\Leftrightarrow$ (ii). On the other hand, using (8) we have

$$
\left(\nabla \pi_{*}\right)(X, B Y)-\left(\nabla \pi_{*}\right)(Y, B X)=-\pi_{*}\left(\nabla_{X} B Y-\nabla_{Y} B X\right) .
$$


Then (6) implies that

$$
\left(\nabla \pi_{*}\right)(X, B Y)-\left(\nabla \pi_{*}\right)(Y, B X)=-\pi_{*}\left(A_{X} B Y-A_{Y} B X\right) .
$$

Since $A_{Y} B X-A_{X} B Y \in \Gamma\left(\left(\operatorname{ker} \pi_{*}\right)^{\perp}\right)$, this shows that (ii) $\Leftrightarrow$ (iii).

Theorem 6. Let $\pi$ be an anti-invariant semi-Riemannian submersion from a para-Kähler manifold $(M, g, P)$ to a semiRiemannian manifold $\left(N, g^{\prime}\right)$. Then the following assertions are equivalent to each other:

(i) $\left(\operatorname{ker} \pi_{*}\right)^{\perp}$ defines a totally geodesic foliation on $M$.

(ii) $g\left(A_{X} B Y, P V\right)=g\left(C Y, P A_{X} V\right)$.

(iii) $g^{\prime}\left(\left(\nabla \pi_{*}\right)(X, B Y), \pi_{*} P V\right)=-g\left(C Y, P A_{X} V\right)$,

for $X, Y \in \Gamma\left(\left(\operatorname{ker} \pi_{*}\right)^{\perp}\right)$ and $V \in \Gamma\left(\operatorname{ker} \pi_{*}\right)$.

Proof. From (1), (2) and (6) we obtain

$$
g\left(\nabla_{X} Y, V\right)=-g\left(A_{X} B Y, P V\right)-g\left(\nabla_{X} C Y, P V\right)
$$

for $X, Y \in \Gamma\left(\left(\operatorname{ker} \pi_{*}\right)^{\perp}\right)$ and $V \in \Gamma\left(\operatorname{ker} \pi_{*}\right)$. Then by (16) we have

$$
g\left(\nabla_{X} Y, V\right)=-g\left(A_{X} B Y, P V\right)+g\left(P A_{X} V, C Y\right)
$$

which shows (i) $\Leftrightarrow$ (ii). On the other hand from (6) and (8) we get

$$
g\left(A_{X} B Y, P V\right)=g^{\prime}\left(-\left(\nabla \pi_{*}\right)(X, B Y), \pi_{*} P V\right) .
$$

This shows (ii) $\Leftrightarrow$ (iii).

Theorem 7. Let $\pi$ be an anti-invariant semi-Riemannian submersion from a para-Kähler manifold $(M, g, P)$ to a semiRiemannian manifold $\left(N, g^{\prime}\right)$. Then the following assertions are equivalent to each other:

(i) $\left(\operatorname{ker} \pi_{*}\right)$ defines a totally geodesic foliation on $M$.

(ii) $g^{\prime}\left(\left(\nabla \pi_{*}\right)(V, P X), \pi_{*} P W\right)=0$.

(iii) $T_{V} B X+A_{C X} V \in \Gamma(\mu)$,

for $X \in \Gamma\left(\left(\operatorname{ker} \pi_{*}\right)^{\perp}\right)$ and $V, W \in \Gamma\left(\operatorname{ker} \pi_{*}\right)$.

Proof. Using (1) and (2) we have $g\left(\nabla_{V} W, X\right)=-g\left(\nabla_{V} P W\right.$, $P X)$. Hence we get $g\left(\nabla_{V} W, X\right)=g\left(h \nabla_{V} P X, P W\right)$. Then a semi-Riemannian submersion $\pi$ and (8) imply that

$$
g\left(\nabla_{V} W, X\right)=-g^{\prime}\left(\left(\nabla \pi_{*}\right)(V, P X), \pi_{*} P W\right)
$$

which is (i) $\Leftrightarrow$ (ii). By direct calculation, we derive

$$
g\left(\nabla_{V} P X, P W\right)=-g^{\prime}\left(\left(\nabla \pi_{*}\right)(V, P X), \pi_{*} P W\right) .
$$

Using (10) we obtain

$$
g\left(\nabla_{V} B X+\nabla_{V} C X, P W\right)=-g^{\prime}\left(\left(\nabla \pi_{*}\right)(V, P X), \pi_{*} P W\right) .
$$

Hence we have

$$
\begin{aligned}
g\left(\nabla_{V} B X+[V, C X]+\nabla_{C X} V, P W\right) \\
=-g^{\prime}\left(\left(\nabla \pi_{*}\right)(V, P X), \pi_{*} P W\right) .
\end{aligned}
$$

Since $[V, C X] \in \Gamma\left(\operatorname{ker} \pi_{*}\right)$, using (4) and (6), we get

$$
\begin{aligned}
& g\left(T_{V} B X+A_{C X} V, P W\right) \\
& \quad=-g^{\prime}\left(\left(\nabla \pi_{*}\right)(V, P X), \pi_{*} P W\right) .
\end{aligned}
$$

This shows (ii) $\Leftrightarrow$ (iii).

We say that an anti-invariant semi-Riemannian submersion is a Lagrangian semi-Riemannian submersion if $P\left(\operatorname{ker} \pi_{*}\right)=\left(\operatorname{ker} \pi_{*}\right)^{\perp}$. If $\mu \neq\{0\}$, then $\pi$ is called a proper anti-invariant semi-Riemannian submersion.

We note that the anti-invariant semi-Riemannian submersion given in Example 3 is a Lagrangian semi-Riemannian submersion.

If $\pi$ is a Lagrangian submersion, then (11) implies that $T N=\pi_{*}\left(P\left(\operatorname{ker} \pi_{*}\right)\right)$.

From Theorem 5 we have the following.

Corollary 8. Let $\pi$ be a Lagrangian semi-Riemannian submersion from a para-Kähler manifold $(M, g, P)$ to a semiRiemannian manifold $\left(N, g^{\prime}\right)$. Then the following assertions are equivalent to each other:

(i) $\left(\operatorname{ker} \pi_{*}\right)^{\perp}$ is integrable.

(ii) $\left(\nabla \pi_{*}\right)(X, P Y)=\left(\nabla \pi_{*}\right)(P X, Y)$.

(iii) $A_{X} P Y=A_{Y} P X$,

for $X, Y \in \Gamma\left(\left(\operatorname{ker} \pi_{*}\right)^{\perp}\right)$.

Theorem 9. Let $\pi$ be a Lagrangian semi-Riemannian submersion from a para-Kähler manifold $(M, g, P)$ to a semiRiemannian manifold $\left(N, g^{\prime}\right)$. Then the following assertions are equivalent to each other:

(i) $\left(\operatorname{ker} \pi_{*}\right)$ defines a totally geodesic foliation on $M$.

(ii) $\left(\nabla \pi_{*}\right)(V, P X)=0$.

(iii) $T_{V} P W=0$,

for $X \in \Gamma\left(\left(\operatorname{ker} \pi_{*}\right)^{\perp}\right)$ and $V, W \in \Gamma\left(\operatorname{ker} \pi_{*}\right)$.

Proof. (i) $\Leftrightarrow$ (ii) is clear from Theorem 7 . We only prove (ii) $\Leftrightarrow$ (iii). From (8), we get

$$
g\left(\nabla_{V} P W, P X\right)=g^{\prime}\left(\left(\nabla \pi_{*}\right)(V, P X), \pi_{*} P W\right)
$$

for $X \in \Gamma\left(\left(\operatorname{ker} \pi_{*}\right)^{\perp}\right)$ and $V, W \in \Gamma\left(\operatorname{ker} \pi_{*}\right)$. Then using (5) we have

$$
g^{\prime}\left(\left(\nabla \pi_{*}\right)(V, P X), \pi_{*} P W\right)=g\left(T_{V} P W, P X\right) .
$$

Since $T_{V} P W \in \Gamma\left(\operatorname{ker} \pi_{*}\right)$, we get (ii) $\Leftrightarrow($ iii).

We note that a differentiable map $\pi$ between two semiRiemannian manifolds is called totally geodesic if $\nabla \pi_{*}=0$. 
Theorem 10. Let $\pi$ be a Lagrangian semi-Riemannian submersion from a para-Kähler manifold $(M, g, P)$ to a semiRiemannian manifold $\left(N, g^{\prime}\right)$. Then $\pi$ is a totally geodesic map if and only if

$$
\begin{gathered}
T_{W} P V=0, \quad \forall W, V \in \Gamma\left(\operatorname{ker} \pi_{*}\right), \\
A_{X} P W=0, \quad \forall X \in \Gamma\left(\left(\operatorname{ker} \pi_{*}\right)^{\perp}\right) .
\end{gathered}
$$

Proof. First of all, we recall that the second fundamental form of a semi-Riemannian submersion satisfies

$$
\left(\nabla \pi_{*}\right)(X, Y)=0, \quad X, Y \in \Gamma\left(\left(\operatorname{ker} \pi_{*}\right)^{\perp}\right) .
$$

For $W, V \in \Gamma\left(\operatorname{ker} \pi_{*}\right)$, by using (2), (5), and (8), we get

$$
\left(\nabla \pi_{*}\right)(W, V)=-\pi_{*}\left(P T_{W} P V\right) .
$$

On the other hand, from (1), (2), and (8), we have

$$
\left(\nabla \pi_{*}\right)(X, W)=-\pi_{*}\left(P \nabla_{X} P W\right)
$$

for $X \in \Gamma\left(\left(\operatorname{ker} \pi_{*}\right)^{\perp}\right)$. Then using (7), we get

$$
\left(\nabla \pi_{*}\right)(X, W)=-\pi_{*}\left(P A_{X} P W\right) .
$$

Since $P$ is nonsingular, proof comes from (37)-(40).

We give a necessary and sufficient condition for a Lagrangian semi-Riemannian submersion to be harmonic.

Theorem 11. Let $\pi$ be a Lagrangian semi-Riemannian submersion from a para-Kähler manifold $(M, g, P)$ to a semiRiemannian manifold $\left(N, g^{\prime}\right)$. Then $\pi$ is harmonic if and only if Trace $P T_{V}=0$ for $V \in \Gamma\left(\operatorname{ker} \pi_{*}\right)$.

Proof. From [23] we know that $\pi$ is harmonic if and only if $\pi$ has minimal fibres. Thus $\pi$ is harmonic if and only if $\sum_{i=1}^{r} T_{e_{i}} e_{i}=0$. On the other hand, from (2), (4), and (5), we obtain

$$
T_{V} P W=P T_{V} W
$$

for any $V, W \in \Gamma\left(\operatorname{ker} \pi_{*}\right)$. Using (41), we get

$$
\sum_{i=1}^{r} g\left(T_{e_{i}} P e_{i}, V\right)=-\sum_{i=1}^{r} g\left(T_{e_{i}} e_{i}, P V\right)
$$

for any $V \in \Gamma\left(\operatorname{ker} \pi_{*}\right)$. Thus using the properties of the O'Neill tensor $T$ we have

$$
\sum_{i=1}^{r} g\left(T_{e_{i}} V, P e_{i}\right)=\sum_{i=1}^{r} g\left(T_{e_{i}} e_{i}, P V\right) .
$$

Since $T$ is symmetric, we obtain

$$
\sum_{i=1}^{r} g\left(T_{V} e_{i}, P e_{i}\right)=\sum_{i=1}^{r} g\left(T_{e_{i}} e_{i}, P V\right) .
$$

Denote by $\left\{L_{i}\right\}$ the canonical foliations on a product manifold $M_{1} \times M_{2}$ with natural projections $p_{i}$ onto $M_{i}$, $(i=1,2)$. Let $P_{i}$ be the projection of $T\left(M_{1} \times M_{2}\right)$ onto $T L_{i}$ and also set $P_{i}^{\perp}=I-P_{i},(i=1,2)$.

Let $\left(M_{i}, g_{i}\right),(i=1,2)$ be semi-Riemannian manifolds and $\lambda_{i}: M_{1} \times M_{2} \rightarrow R$ be positive differentiable functions. A double-twisted product $M_{1} \times{ }_{\left(\lambda_{1}, \lambda_{2}\right)} M_{2}$ is the differentiable manifold $M_{1} \times M_{2}$ with a semi-Riemannian metric defined by

$$
g(X, Y)=\lambda_{1}^{2} g_{1}\left(P_{1} X, P_{1} Y\right)+\lambda_{2}^{2} g_{2}\left(P_{2} X, P_{2} Y\right)
$$

for all vectors $X$ and $Y$ tangent to $M_{1} \times M_{2}$. In particular, if $\lambda_{1}=1$ then ${ }_{1} M_{1} \times{ }_{\lambda_{2}} M_{2}=M_{1} \times{ }_{\lambda_{2}} M_{2}$ is called the twisted product of $\left(M_{2}, g_{2}\right)$ with twisting function $\lambda_{2}$. Moreover, if $\lambda_{2}$ only depends on the points of $M_{1}$ then $M_{1} \times{ }_{\lambda_{2}} M_{2}$ is called the warped product of $\left(M_{1}, g_{1}\right)$ and $\left(M_{2}, g_{2}\right)$ with warping function $\lambda_{2}$.

In [24], the relations between the twisted and warped product structures in semi-Riemannian geometry are studied. There, essentially the following is proven (cf. [24], Proposition 3).

Let $g$ be a semi-Riemannian metric tensor on the manifold $M=M_{1} \times M_{2}$ and assume that the canonical foliations $L_{1}$ and $L_{2}$ intersect perpendicularly everywhere. Then $g$ is the metric tensor of

(1) a twisted product $M_{1} \times{ }_{\lambda} M_{2}$ if $L_{1}$ is a totally geodesic foliation and $L_{2}$ is a totally umbilic foliation,

(2) a warped product $M_{1} \times{ }_{\lambda} M_{2}$ if $L_{1}$ is a totally geodesic foliation and $L_{2}$ is a spheric foliation; that is, it is umbilical and its mean curvature vector field is parallel,

(3) a usual product of semi-Riemannian manifolds if and only if $\mathrm{f} L_{1}$ and $L_{2}$ are totally geodesic foliations.

Now, we obtain decomposition theorems by using the existence of anti-invariant semi-Riemannian submersions.

Theorem 12. Let $(M, g, P)$ be a para-Kähler manifold and $\left(N, g^{\prime}\right)$ be a semi-Riemannian manifold. If there exists a Lagrangian semi-Riemannian submersion from $M$ onto $N$ such that $M$ is locally twisted product manifold of the form $M_{\mathrm{ker} \pi_{*}} \times_{f} M_{\left(\mathrm{ker} \pi_{*}\right)^{\perp}}$, then $M$ is a usual (locally product) manifold, where $M_{\left(\mathrm{ker} \pi_{*}\right)^{\perp}}$ and $M_{\mathrm{ker} \pi_{*}}$ are integral manifolds of the distribution $\left(\operatorname{ker} \pi_{*}\right)^{\perp}$ and $\operatorname{ker} \pi_{*}$.

Proof. Suppose that $\pi:(M, g, P) \rightarrow\left(N, g^{\prime}\right)$ is a Lagrangian semi-Riemannian submersion and $M$ is a locally twisted product of the form $M_{\mathrm{ker} \pi_{*}}{ }_{f} M_{\left(\mathrm{ker} \pi_{*}\right)^{\perp}}$. Then $M_{\mathrm{ker} \pi_{*}}$ is a totally geodesic foliation and $M_{\left(\operatorname{ker} \pi_{*}\right)^{\perp}}$ is a totally umbilical foliation. We denote the second fundamental form of $M_{\left(\operatorname{ker} \pi_{*}\right)^{\perp}}$ by $h$. Then we get $g\left(\nabla_{X} Y, V\right)=g(h(X, Y), V)$ for $X, Y \in \Gamma\left(\left(\operatorname{ker} \pi_{*}\right)^{\perp}\right.$ and $V \in \Gamma\left(\operatorname{ker} \pi_{*}\right)$. Since $M_{\left(\operatorname{ker} \pi_{*}\right)^{\perp}}$ is totally umbilical we have

$$
g\left(\nabla_{X} Y, V\right)=g(H, V) g(X, Y),
$$

where $H$ is the mean curvature vector field of $M_{\left(\operatorname{ker} \pi_{*}\right)^{\perp}}$. On the other hand, from (2), we derive $g\left(\nabla_{X} Y, V\right)=$ $g\left(\nabla_{X} P V, P Y\right)$. Then using (7), we obtain

$$
g\left(\nabla_{X} Y, V\right)=g\left(A_{X} P V, P Y\right) .
$$


Thus, from (46) and (47), we have

$$
A_{X} P V=-g(H, V) P X
$$

Hence, we arrive at

$$
g\left(A_{X} P V, P X\right)=g(H, V) g(X, X) .
$$

Then using (7) we get

$$
g\left(\nabla_{X} P V, P X\right)=g(H, V) g(X, X) .
$$

Thus (2) implies that

$$
-g\left(\nabla_{X} V, X\right)=g(H, V) g(X, X) .
$$

Hence, we obtain

$$
g\left(\nabla_{X} X, V\right)=g(H, V) g(X, X) .
$$

Then, since $A$ is alternating on the horizontal distribution, we have $A_{X} X=0$ which implies that $g(H, V) g(X, X)=0$. Since $g$ is a semi-Riemannian metric and $H \in \Gamma\left(\operatorname{ker} \pi_{*}\right)$, we conclude that $H=0$. This shows that $\left(\operatorname{ker} \pi_{*}\right)^{\perp}$ is totally geodesic, so $M$ is usual product of Riemannian manifolds. Thus the proof is complete.

Theorem 13. Let $\pi$ be a Lagrangian semi-Riemannian submersion from a para-Kähler manifold $(M, g, P)$ to a semiRiemannian manifold $\left(N, g^{\prime}\right)$. Then $M$ is a locally twisted product manifold of the form $M_{\left(\operatorname{ker} \pi_{*}\right)} \times_{f} M_{\mathrm{ker} \pi_{*}}$ if and only if

$$
T_{V} P X=-g\left(X, T_{V} V\right) g(V, V)^{-1} P V
$$

for $X, Y \in \Gamma\left(\left(\operatorname{ker} \pi_{*}\right)^{\perp}\right)$ and $V \in \Gamma\left(\operatorname{ker} \pi_{*}\right)$, where $\left.M_{\left(\operatorname{ker} \pi_{*}\right)}\right)^{\perp}$ and $M_{\mathrm{ker} \pi_{*}}$ are integral manifolds of the distribution $\left(\operatorname{ker} \pi_{*}\right)^{\perp}$ and $\operatorname{ker} \pi_{*}$.

Proof. From (2) and (5), we obtain

$$
g\left(\nabla_{V} W, X\right)=-g\left(T_{V} P W, P X\right)
$$

for $X \in \Gamma\left(\left(\operatorname{ker} \pi_{*}\right)^{\perp}\right)$ and $V, W \in \Gamma\left(\operatorname{ker} \pi_{*}\right)$. Since $T_{V}$ is skewsymmetric, we get

$$
g\left(\nabla_{V} W, X\right)=g\left(T_{V} P X, P W\right) .
$$

This implies that ker $\pi_{*}$ is totally umbilical if and only if

$$
X(\lambda) P V=T_{V} P X
$$

where $\lambda$ is a function on $M$. Then by direct computations, it is easy to see that this is equivalent to

$$
T_{V} P X=-g\left(X, T_{V} V\right) g(V, V)^{-1} P V .
$$

Thus the proof is complete.

\section{Curvature Relations for Anti-Invariant Semi-Riemannian Submersions}

In this section, we are going to obtain curvature relations of anti-invariant semi-Riemannian submersions.

Let $(M, g)$ be a semi-Riemannian manifold. The sectional curvature $K$ of a 2-plane in $T_{p} M, p \in M$, spanned by $\{X, Y\}$, is defined by

$$
K(X, Y)=\frac{R(X, Y, X, Y)}{g(X, X) g(Y, Y)-g(X, Y)^{2}} .
$$

It is clear that the above definition makes sense only for nondegenerate planes, that is, those satisfying $Q(X, Y)=$ $g(X, X) g(Y, Y)-g(X, Y)^{2} \neq 0$.

Lemma 14. Let $\pi$ be an anti-invariant semi-Riemannian submersion from a para-Kähler manifold $(M, g, P)$ to a semi-Riemannian manifold $\left(N, g^{\prime}\right)$. Then for $X, Y \in \Gamma\left(\left(\operatorname{ker} \pi_{*}\right)^{\perp}\right)$ and $U, V \in \Gamma\left(\operatorname{ker} \pi_{*}\right)$, we have the following relations:

$$
\begin{gathered}
B T_{U} V=T_{U} P V ; \\
h \nabla_{U} P V=C T_{U} V+P \widehat{\nabla}_{U} V ; \\
P A_{X} Y+C h \nabla_{X} Y=A_{X} B Y+h \nabla_{X} C Y ; \\
B h \nabla_{X} Y=v \nabla_{X} B Y+A_{X} C Y ; \\
B A_{X} U=A_{X} P U .
\end{gathered}
$$

Proof. From (2) and (5) we have

$$
P \nabla_{U} V=h \nabla_{U} P V+T_{U} P V
$$

Using (4) we get

$$
P T_{U} V+P \widehat{\nabla}_{U} V=h \nabla_{U} P V+T_{U} P V
$$

Then (10) implies that

$$
B T_{U} V+C T_{U} V+P \widehat{\nabla}_{U} V=h \nabla_{U} P V+T_{U} P V .
$$

Taking the vertical and horizontal parts of this equation, we obtain (59) and (60). The other assertions can be obtained in a similar way.

Using both Lemma 14 and pages 13-14 of [21], we obtain the following.

Theorem 15. Let $\pi$ be an anti-invariant semi-Riemannian submersion from a para-Kähler manifold $(M, g, P)$ to a semiRiemannian manifold $\left(N, g^{\prime}\right)$. Let $U$ and $V$ be nonzero nonlightlike orthogonal unit vertical vectors. Then, we have

$$
\begin{gathered}
K(U \wedge V) \\
=K^{*}(P U \wedge P V)+\epsilon_{U} \epsilon_{V}\left[3 g\left(A_{P U} V, A_{P U} V\right)\right. \\
\left.+3 g\left(C A_{P U} V, C A_{P U} V\right)\right] \\
H(U)=-\left(g\left(\left(\nabla_{P U} T\right)_{U} U, P U\right)+g\left(T_{U} U, T_{U} U\right)\right. \\
\left.+g\left(C T_{U} U, C T_{U} U\right)+g\left(A_{P U} U, A_{P U} U\right)\right),
\end{gathered}
$$


where $\epsilon_{U}=g(U, U) \in\{ \pm 1\}, \epsilon_{V}=g(V, V) \in\{ \pm 1\}$ and $H(U)=$ $K(U \wedge P U)$ is the para-holomorphic sectional curvature of $M$.

From Theorem 15 we have the following result.

Corollary 16. Let $\pi$ be an anti-invariant semi-Riemannian submersion from a para-Kähler manifold $(M, g, P)$ to a semiRiemannian manifold $\left(N, g^{\prime}\right)$. Let $U$ and $V$ be nonzero nonlightlike orthogonal unit vertical vectors. Then, $K(U \wedge$ $V)=K^{*}(P U \wedge P V)$ if and only if $g\left(A_{P U} V, A_{P U} V\right)=$ $-g\left(C A_{P U} V, C A_{P U} V\right)$, where $K^{*}$ is the sectional curvature of $N$.

\section{Conflict of Interests}

The author declares that he has no conflict of interests.

\section{Acknowledgment}

The author is grateful to the referee(s) for their valuable comments and suggestions.

\section{References}

[1] B. O'Neill, "The fundamental equations of a submersion," The Michigan Mathematical Journal, vol. 13, pp. 459-469, 1966.

[2] A. Gray, "Pseudo-Riemannian almost product manifolds and submersions," Journal of Applied Mathematics and Mechanics, vol. 16, pp. 715-737, 1967.

[3] B. Watson, "Almost Hermitian submersions," Journal of Differential Geometry, vol. 11, no. 1, pp. 147-165, 1976.

[4] D. Chinea, "Almost contact metric submersions," Rendiconti del Circolo Matematico di Palermo, vol. 34, no. 1, pp. 89-104, 1985.

[5] S. Ianuş, R. Mazzocco, and G. E. Vîlcu, "Riemannian submersions from quaternionic manifolds," Acta Applicandae Mathematicae, vol. 104, no. 1, pp. 83-89, 2008.

[6] A. V. Caldarella, "On paraquaternionic submersions between paraquaternionic Kähler manifolds," Acta Applicandae Mathematicae, vol. 112, no. 1, pp. 1-14, 2010.

[7] S. Ianuş, S. Marchiafava, and G. E. Vîlcu, "Paraquaternionic CR-submanifolds of paraquaternionic Kähler manifolds and semi-Riemannian submersions," Central European Journal of Mathematics, vol. 8, no. 4, pp. 735-753, 2010.

[8] Y. Gündüzalp and B. Şahin, "Paracontact semi-Riemannian submersions," Turkish Journal of Mathematics, vol. 37, no. 1, pp. 114-128, 2013.

[9] Y. Gündüzalp and B. Şahin, "Para-contact para-complex semiRiemannian submersions," Bulletin of the Malaysian Mathematical Sciences Society. In press.

[10] Y. Gündüzalp, "Slant submersions from almost product Riemannian manifolds," Turkish Journal of Mathematics, vol. 37, no. 5, pp. 863-873, 2013.

[11] K.-S. Park, " $H$-slant submersions," Bulletin of the Korean Mathematical Society, vol. 49, no. 2, pp. 329-338, 2012.

[12] B. Şahin, "Slant submersions from almost Hermitian manifolds," Bulletin Mathématique de la Société des Sciences, vol. 54, no. 102, pp. 93-105, 2011.

[13] B. Şahin, "Anti-invariant Riemannian submersions from almost Hermitian manifolds," Central European Journal of Mathematics, vol. 8, no. 3, pp. 437-447, 2010.
[14] B. Şahin, "Anti-invariant riemannian submersions from almost hermitian manifolds: curvatures," in Proceedings of the 1st International Eurasian Conference on Mathematical Sciences and Applications, pp. 3-7, Prishtine, Kosovo, September 2012.

[15] P. Libermann, "Sur les structures presque paracomplexes," Comptes Rendus de l'Académie des Sciences, vol. 234, pp. 25172519, 1952.

[16] P. K. Raševskiŭ, “The scalar field in a stratified space," Trudy Seminara po Vektornomu i Tenzornomu Analizu, vol. 6, pp. 225248, 1948.

[17] B. O'Neill, Semi-Riemannian Geometry with Applications to Relativity, vol. 103, Academic Press, New York, NY, USA, 1983.

[18] M. Falcitelli, S. Ianus, A. M. Pastore, and M. Visinescu, "Some applications of Riemannian submersions in physics," Revue Roumaine de Physique, vol. 48, pp. 627-639, 2003.

[19] S. Ivanov and S. Zamkovoy, "Para-Hermitian and paraquaternionic manifolds," Differential Geometry and Its Applications, vol. 23, pp. 205-234, 2005.

[20] G. Bădiţoiu and S. Ianuş, "Semi-Riemannian submersions from real and complex pseudo-hyperbolic spaces," Differential Geometry and its Applications, vol. 16, no. 1, pp. 79-94, 2002.

[21] M. Falcitelli, S. Ianus, and A. M. Pastore, Riemannian Submersions and Related Topics, World Scientific, River Edge, NJ, USA, 2004.

[22] S. Ianuş, G. E. Vîlcu, and R. C. Voicu, "Harmonic maps and Riemannian submersions between manifolds endowed with special structures," in Algebra, Geometry and Mathematical Physics, vol. 93, pp. 277-288, Banach Center Publications, 2011.

[23] J. Eells, Jr. and J. H. Sampson, "Harmonic mappings of Riemannian manifolds," American Journal of Mathematics, vol. 86, pp. 109-160, 1964.

[24] R. Ponge and H. Reckziegel, "Twisted products in pseudoRiemannian geometry," Geometriae Dedicata, vol. 48, no. 1, pp. 15-25, 1993. 


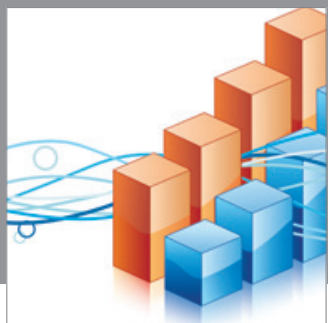

Advances in

Operations Research

mansans

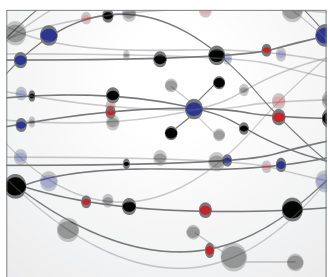

The Scientific World Journal
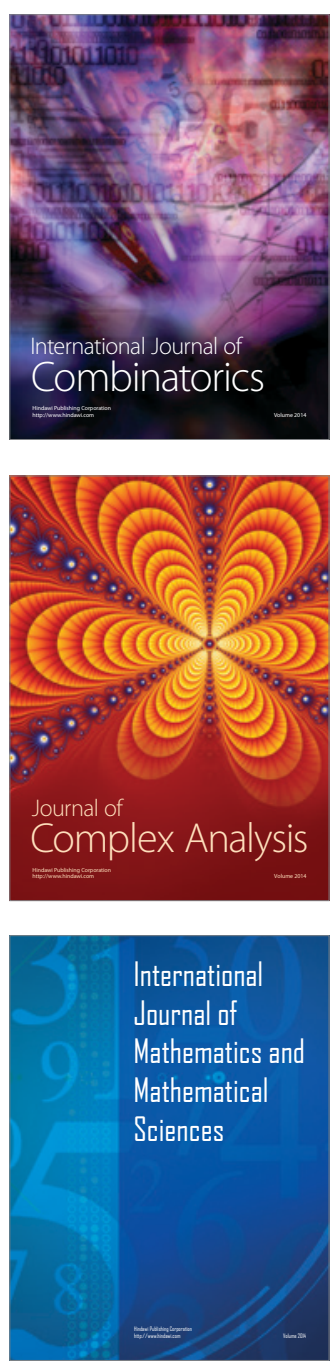
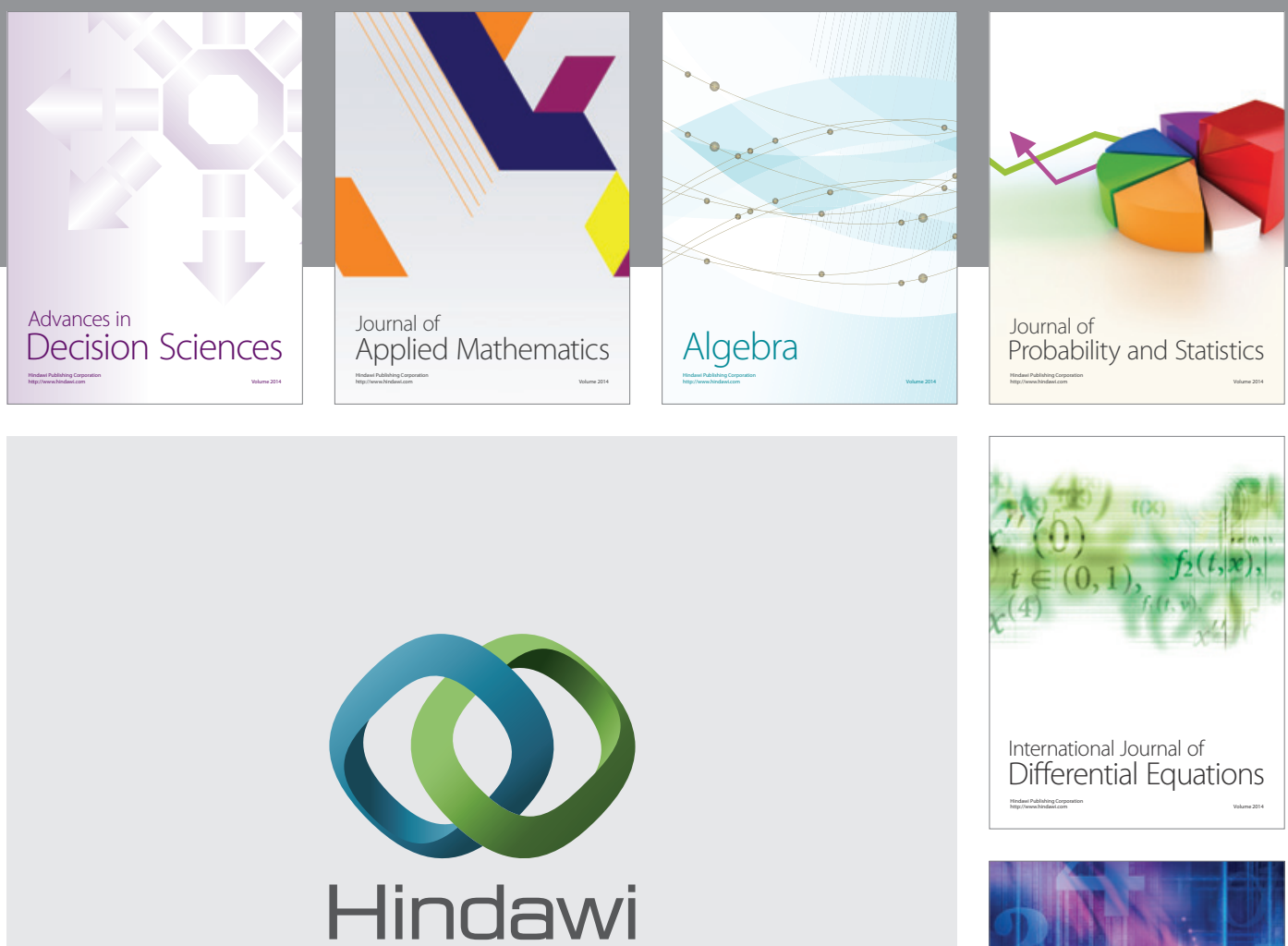

Submit your manuscripts at http://www.hindawi.com
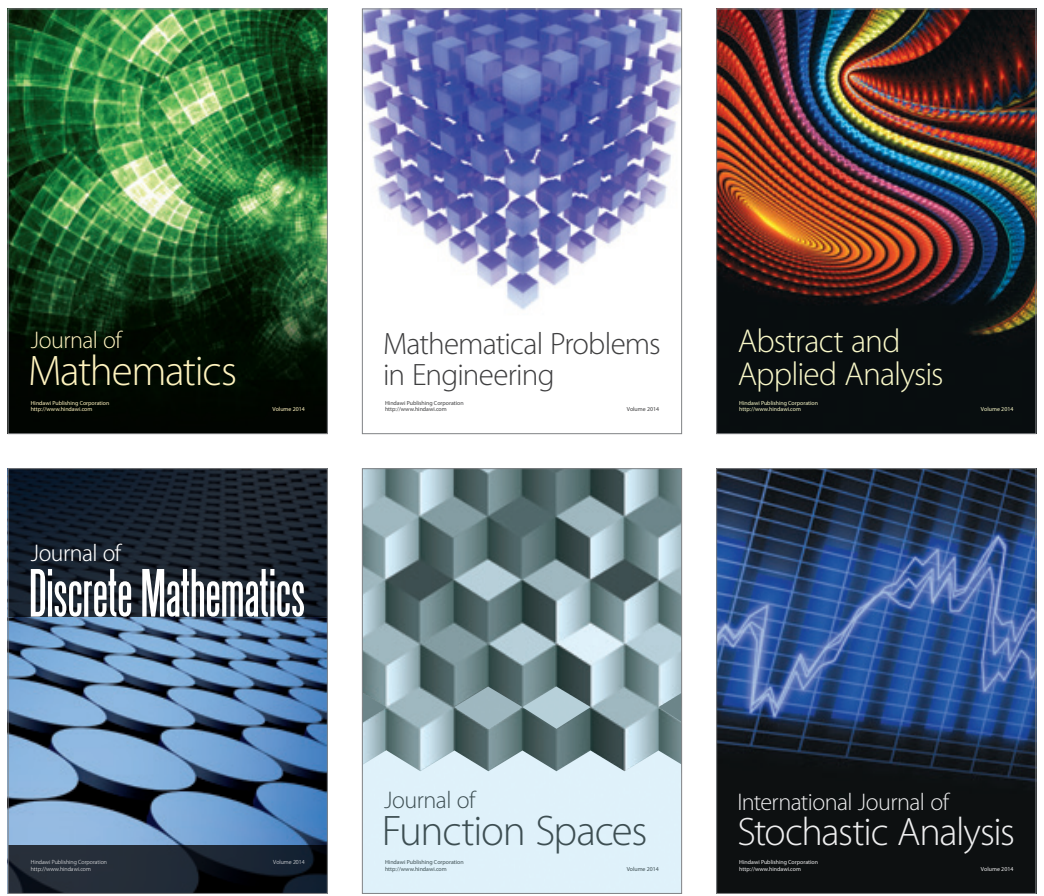

Journal of

Function Spaces

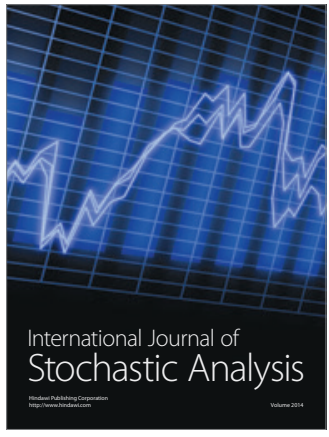

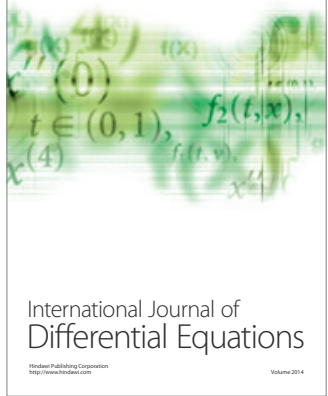
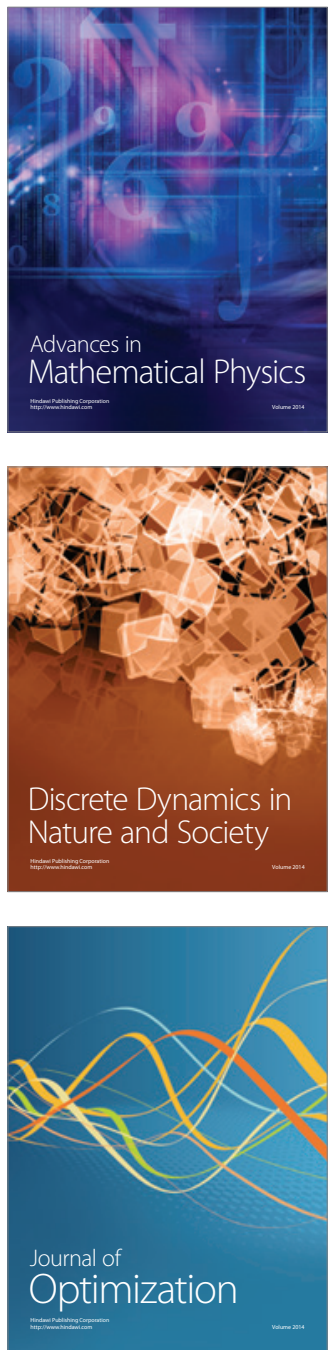